\title{
Genetic epidemiology of Sarcoptes scabiei in the Iberian wolf in Asturias, Spain
}

\author{
Alvaro Oleaga ${ }^{\mathrm{a}, \mathrm{b}, *, 1}$, Samer Alasaad ${ }^{\mathrm{c}, \mathrm{d}, \mathrm{e}, 1}$, Luca Rossi $^{\mathrm{e}}$, Rosa Casais ${ }^{\mathrm{f}}$, \\ Joaquin Vicente ${ }^{\mathrm{a}}$, Sandra Maione ${ }^{\mathrm{e}}$, Ramón C. Soriguer ${ }^{\mathrm{d}}$, Christian Gortázar ${ }^{\mathrm{a}}$ \\ a Instituto de Investigación en Recursos Cinegéticos IREC (CSIC-UCLM-JCCM). Ronda de Toledo, s/n 13071, Ciudad Real, Spain \\ b SERPA, Sociedad de Servicios del Principado de Asturias S.A., 33203 Gijón, Asturias, Spain \\ c Institute of Evolutionary Biology and Environmental Studies (IEU), University of Zürich, Winterthurerstrasse 190, 8057 Zürich, \\ Switzerland \\ d Estación Biológica de Doñana, Consejo Superior de Investigaciones Científicas (CSIC), Avda. Américo Vespucio, s/n 41092 Sevilla, Spain \\ e Dipartimento di Scienzr veterinarie, Università degli Studi di Torino, Via Leonardo da Vinci 44, I-10095 Grugliasco, Italy \\ f SERIDA, Servicio Regional de Investigación y Desarrollo Agroalimentario, Centro de Biotecnología Animal, Camino de Rioseco 1225, \\ 33394 Deva-Gijón, Asturias, Spain
}

Keywords:

Sarcoptic mange

Canis lupus

Microsatellites

Genetic structure

Gene flow

Prey-to-predator

Host taxon-derived

\section{A B S T R A C T}

Background: During the last decades, attempts have been made to understand the molecular epidemiology of Sarcoptes scabiei, and to detect and clarify the differences between isolates from different hosts and geographic regions. Two main phenomena have been described: (i) host-taxon derived-Sarcoptes mite infection in European wild animals (revealing the presence of three separate clusters, namely herbivore-, carnivore- and omnivore-derived Sarcoptes populations in Europe) and (ii) prey-to-predator Sarcoptes mite infection in the Masai Mara ecosystem.

Results: Using one multiplex of 9 microsatellite markers and Sarcoptes mite samples from sympatric Pyrenean chamois, red deer, red fox and Iberian wolf, different population structure analyses revealed concordance with the host-taxon law described for wild animals in Europe, with two main host-derived Sarcoptes mite populations, herbivore- and carnivorederived. Surprisingly, Iberian wolf derived Sarcoptes populations had the highest genetic diversity among the other populations, including two different subpopulations: one similar to the herbivore-derived Sarcoptes populations, and another similar to carnivore (fox)derived Sarcoptes mite population.

Conclusions: The host-taxon effect in wild animals is still supported with the maintenance of carnivore- and herbivore-derived Sarcoptes clusters' separation in analyzed mites. However, this phenomenon could be modified with the inclusion of a large predator as wolf in the present work, revealing prey-to-predator Sarcoptes mite infection between the studied host-taxa and suggesting the importance of wolf's immune system for explaining the high variability reported in C. lupus derived mites. Further studies of host diet, behavior and movement, and regarding the role played by its immune system, would be of great help to clarify interactions between the two hypotheses, host-taxon and prey-to-predator.

\footnotetext{
* Corresponding author at: SERIDA, Servicio Regional de Investigación y Desarrollo Agroalimentario, Centro de Biotecnología Animal, Camino de Rioseco 1225, 33394 Deva-Gijón, Asturias, Spain. Tel.: +34 926 295450; fax: +34926 295451

E-mail addresses: alvaroleaga@yahoo.es, alvaro.oleaga@uclm.es (A. Oleaga).

1 Contributed equally.
}

\section{Introduction}

Despite representing the first human illness with a known etiologic agent (Montesu et al., 1991; Gakuya et al., 2012a) and affecting more than 100 mammal species 
all over the world (Bornstein et al., 2001; Pence and Ueckerman, 2002), the taxonomical status of the ectoparasitic burrowing mite Sarcoptes scabiei has been the subject of continuous debate for decades (Pence et al., 1975; Fain, 1978; Andrews, 1983). The development of molecular techniques has provided new approaches and novel data on this subject (Zahler et al., 1999; Walton et al., 2004a; Gu and Yang, 2008); in particular, microsatellites are a valuable technique for the analysis of $S$. scabiei and the relationship between hosts. Microsatellite analysis supports the standing of S. scabiei as a single highly variable species with different strains manifesting physiological host-specificity (Walton et al., 2004b; Alasaad et al., 2008b, 2011b, 2012).

A mange epizootic first detected in 1993 in the southern border of Asturias severely affected Southern chamois (Rupicapra pyrenaica parva) from the area (FernándezMoran et al., 1997), leading to an overall $61.3 \%$ reduction of their population during the first years after the outbreak (González-Quirós et al., 2002). Although the origin of the outbreak could not be demonstrated, it has been attributed to mangy domestic goats sharing pastures with chamois in the study area, with subsequent cross-infection (Lavín et al., 2000; Menzano et al., 2007). The epizootic is still expanding eastwards today with sporadic cases of sarcoptic mange in chamois, red deer (Cervus elaphus) and roe deer (Capreolus capreolus - Oleaga et al., 2008a,b) from the area. Red foxes (Vulpes vulpes) and wolves (Canis lupus) have also been diagnosed with sarcoptic mange in Western, Central and Eastern Regions of Asturias, and disease is now considered endemic in red foxes.

Three main groups of S. scabiei mites have been genetically identified in European wildlife studied so far, namely herbivore, carnivore and omnivore-derived Sarcoptes clusters (Rasero et al., 2010). These mite "strains" occur in sympatric as well as geographically distant populations, including Southern chamois, red deer, roe deer and red fox in our study area in Asturias, Northern Spain (Alasaad et al., 2011b). In sympatric mammals, differentiation of mites into "strains" may result from null or limited interspecific transmission, due to low frequency of direct contacts among hosts, low densities of one or more hosts, or different immunological response to the invading mites, limiting their population size, hence their number on the skin surface and their transmissibility to other individuals of the same or a different species. On the other hand, genetically based preference for a particular host (leading to hostassociated mating) or the evolution of higher performance or viability on that host (usually entailing a worse performance on the others, Kassen, 2002) have been proposed as possible mite-dependent mechanisms limiting gene flow (Magalhães et al., 2007). The apparent lack of gene flow or recent admixture between carnivore-, herbivore-, and omnivore-derived Sarcoptes populations reported in European studied species, including sympatric animals, led to the so called "host-taxon law" formulation (Rasero et al., 2010; Alasaad et al., 2011b).

Parasites are of pivotal importance in food webs. Moreover food webs are very incomplete without parasites (Lafferty et al., 2006), with endo-parasites (Sukhdeo, 2012) as the most frequently described trophically transmitted parasites (Luong et al., 2013). The only report, to our knowledge, about ecto-parasites transmission was on Sarcoptes mite transmission in Masai Mara ecosystem (Gakuya et al., 2011). Theoretically, the higher frequency of direct contacts among hosts within trophic chains (especially when acting as predator, considering that prey are not a "longlasting available habitat" after predation for ectoparasitic mites) should favor a more efficient gene flow between different mite "strains". Nevertheless, the study of $S$. scabiei molecular features in a predator/prey ecosystem has not been carried out in any European model, so far. Recently, molecular and epidemiological studies including wild felids and ungulates in Africa have signaled the existence of a potential predator/prey bond in sarcoptic mange transmission, the so called prey-to-predator parasitic infection (Gakuya et al., 2011, 2012b).

The recent isolation of $S$. scabiei mites in 9 out of 12 wolves bearing skin lesion in Asturias (Northern Spain) suggested an increase in morbidity of sarcoptic mange in this wild canid population (Domínguez et al., 2008; Oleaga et al., 2011) and allowed a genetic study of mites in this host for the first time. This work aims to study the genetic structure of S. scabiei mites affecting wolves in Northern Spain, and to genetically compare them with mites originating from sympatric wild herbivores (red deer and Southern chamois) and carnivores (red fox). Both wild and domestic ungulates are common prey of the wolf in the study area (Meriggi and Lovari, 1996; Barja, 2009), whereas predation and scavenging on mangy foxes have been suspected as a source of infection in this top chain carnivore (Bornstein et al., 1995; Mörner et al., 2005; Domínguez et al., 2008). Such molecular analyses could hep in determining the extent to which sarcoptic mites may be interspecifically transmitted in one of the few available large predator-prey food chains in Europe.

\section{Materialsandmethods}

\subsection{Study area}

The study was carried out in the Principality of Asturias, a $10,603 \mathrm{~km}^{2}$ autonomous region located in North-Western Spain. The area, with a mixture of open pastures and meadows with deciduous and mixed forests, is home of many different wildlife species, including carnivores like the endangered brown bear (Ursus arctos), wolf and red fox, and several wild ungulates (red deer, roe deer, wild boar Sus scrofa - and Cantabrian chamois) that represent a high percentage of the diet of wolves (Llaneza et al., 1996).

\subsection{Sampling and mite isolation}

The study of wolves submitted for necropsy in Asturias (Northern Spain) as part of a wildlife diseases surveillance program allowed the confirmation of sarcoptic mange by mite isolation in 9 out of 47 wolves from 2008 to 2010 (Oleaga et al., 2011), and the extraction of genetic material from mites belonging to 8 of these 9 mangy wolves (seven of them were collected in population control hunts carried out by wildlife officers, while one wolf died as a consequence of vehicle collision). Acari were collected after 
incubation of 8 skin pieces from each animal on Petri dishes for $24 \mathrm{~h}$ at $37^{\circ} \mathrm{C}$ and meticulous examination for the detection and identification of ectoparasites using an Olympus SZX9 (10-57×) magnifier (Alasaad et al., 2009). Collected mites were identified as $S$. scabiei according to Wall and Shearer (1997) and preserved in 70\% ethanol until DNA extraction. DNA was extracted from 19 mites belonging to 8 mangy confirmed wolves (seven adults and two 6 month old pups).

Mites obtained from 7 chamois $(n=14), 8$ red deer $(n=13)$ and 6 red foxes $(n=12)$ with confirmed sarcoptic mange were also collected in Asturias from 2006 to 2010, and analyzed in order to compare molecular features with those reported in Iberian wolves.

\subsection{DNA extraction and fluorescent-based polymerase chain reaction (PCR) analysis of microsatellite DNA}

The DNA of individual Sarcoptes mites was extracted using the HotSHOT Plus ThermalSHOCK technique (Alasaad et al., 2008a). Two blanks (reagents only) were included in each extraction to monitor for contamination.

As described by Alasaad et al. (2008b), nine specific Sarcoptes mite microsatellites (Sarms 33-38, 40, 41, and 44) were used with one $9 \times$ multiplex PCR. One primer from each set was $5^{\prime}$ labeled with 6-FAM, VIC, NED or PET ${ }^{\circledR}$ fluorescent dye tag (Applied Biosystems, Foster City, CA, USA). Each $15 \mu$ l PCR reaction mixture consisted of $3 \mu$ l of the single mite DNA, together with the PCR mixture containing all primer pairs (ranging from 0.04 to $0.1 \mu \mathrm{M}$ per primer), $200 \mu \mathrm{M}$ of each dNTP, $1.5 \mu \mathrm{l}$ of $10 \times$ PCR buffer $(200 \mathrm{mM} \mathrm{KCl}$ and $100 \mathrm{mM}$ Tris- $\mathrm{HCl}, \mathrm{pH} 8.0), 1.5 \mathrm{mM} \mathrm{MgCl}_{2}$ and $0.15 \mu \mathrm{l}$ (0.5 U/reaction) HotStar Taq (QIAGEN, Milano, Italy). The thermal profile in a 2720 thermal cycler (Applied Biosystems, Foster City, CA, USA) was as follows: $15 \mathrm{~min}$ at $95^{\circ} \mathrm{C}$ (initial denaturing), followed by 37 cycles of three steps of $30 \mathrm{~s}$ at $94^{\circ} \mathrm{C}$ (denaturation), $45 \mathrm{~s}$ at $55^{\circ} \mathrm{C}$ (annealing) and $1.5 \mathrm{~min}$ at $72^{\circ} \mathrm{C}$ (extension), before a final elongation of $7 \mathrm{~min}$ at $72^{\circ} \mathrm{C}$. Fluorescent PCR amplification products were analyzed using formamide with Size Standard $500 \mathrm{Liz}$ (Applied Biosystems, Foster City, CA, USA) by ABI PRISM 310 Genetic Analyser with pop4. Allele calling was performed using the GeneMapper v. 4.0 software (Applied Biosystems, Foster City, CA, USA). Possible genotyping mistakes (scoring error due to stuttering, large allele dropout) were estimated using MICROCHECKER (Oosterhout et al., 2004).

\subsection{Molecular analyses}

Expected $\left(H_{E}\right)$ and observed $\left(H_{O}\right)$ heterozygosity, linkage disequilibria (LD), and HWE tests were calculated using GENEPOP (v.3.4 [Raymond and Rousset, 1995]). Deviations from HWE and tests for LD were evaluated using Fisher's exact tests and sequential Bonferroni corrections.

The heterogeneity of genetic diversity among the different Sarcoptes mite populations was estimated by the partition of variance components (AMOVA) applying conventional $F_{S T}$ statistics using allele's frequencies as implemented in Arlequin 3.11 (Excoffier, 2006). The analysis of relationships between mites was carried out by the
Bayesian assignment test of the software STRUCTURE (v.2.3.3 [Pritchard et al., 2000]). Burn-in and run lengths of Markov chains were both 100,000 . We ran 20 independent runs for each $K$ (for $K=1-10$ ). The most likely number of clusters was determined using two approaches; by estimating the posterior probability for each $K$, using the method of Evanno et al. (2005). Finally, each of the inferred clusters was associated with the component populations of its mites.

The degree of genetic relationship among populations was further investigated with FCA as implemented in Genetix v.4.05.2 (Belkhir, 1999).

\section{Results}

Twenty-eight alleles were detected in the 9 microsatellite loci studied in wolf derived-Sarcoptes mites, including one private allele (present only in the wolf population, Sarms 44). The number of alleles for each locus ranged in the wolf population from two (Sarms40 and Sarms 41) to four (Sarms35, Sarms37 and Sarms38). All the alleles detected in studied ungulate species $(n=12$, with 11,12

\section{Table1}

Comparison of allele size and total number of detected alleles in the four sympatric species-derived mite populations studied in Asturias at the 9 microsatellite loci. Private alleles are shown in black, while alleles present both in ungulates and red fox (that are also present in wolf) are underlined and in italics.

\begin{tabular}{|c|c|c|c|c|}
\hline Locus\# & Chamois & Red deer & Red fox & Wolf \\
\hline \multirow[t]{3}{*}{ Sarms33 } & 226 & 226 & 232 & 226 \\
\hline & & & 240 & 232 \\
\hline & & & & 240 \\
\hline \multirow[t]{3}{*}{ Sarms34 } & 176 & 176 & & 176 \\
\hline & 198 & 198 & & 198 \\
\hline & & & 174 & 174 \\
\hline \multirow[t]{4}{*}{ Sarms35 } & 162 & 162 & & 162 \\
\hline & & & 148 & 148 \\
\hline & & & 152 & 152 \\
\hline & & & 156 & 156 \\
\hline \multirow[t]{3}{*}{ Sarms36 } & 279 & 279 & 279 & $\underline{279}$ \\
\hline & & 281 & 281 & $\underline{281}$ \\
\hline & & & 283 & 283 \\
\hline \multirow[t]{4}{*}{ Sarms37 } & 172 & 172 & & 172 \\
\hline & & & 164 & 164 \\
\hline & & & 170 & 170 \\
\hline & & & 178 & 178 \\
\hline \multirow[t]{4}{*}{ Sarms38 } & 213 & 213 & & 213 \\
\hline & 215 & 215 & & 215 \\
\hline & & & 209 & 209 \\
\hline & & & 211 & 211 \\
\hline \multirow[t]{2}{*}{ Sarms40 } & 215 & 215 & & 215 \\
\hline & & & 217 & 217 \\
\hline \multirow[t]{2}{*}{ Sarms41 } & 236 & 236 & & 236 \\
\hline & & & 234 & 234 \\
\hline \multirow[t]{2}{*}{ Sarms44 } & 262 & 262 & 262 & $\underline{262}$ \\
\hline & & & 270 & $\overline{270}$ \\
\hline
\end{tabular}

Total number of alleles

11

12

20 


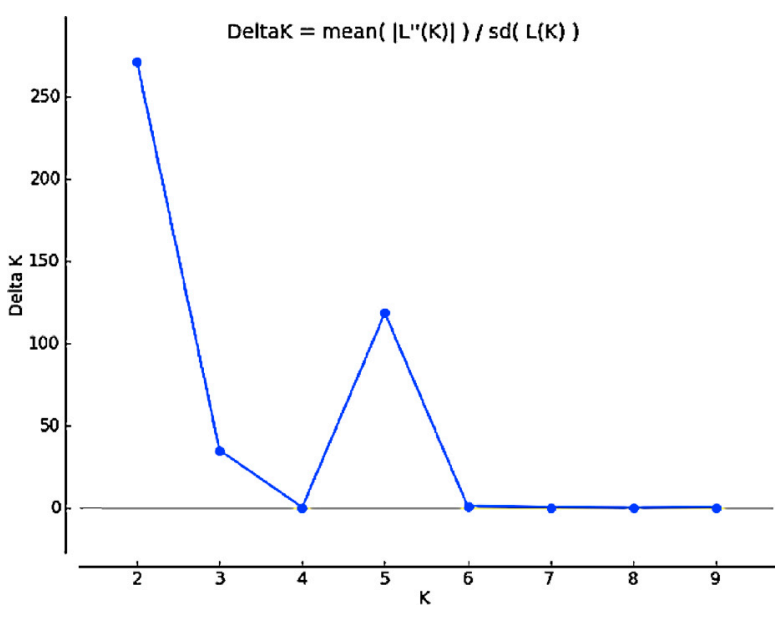

Fig.1. Results of STRUCTURE analysis showing $\Delta K$ as proposed by Evanno et al. (2005) method. The best fit of the data was two clusters.

and 9 alleles for chamois, red deer and roe deer, respectively, Alasaad et al., 2011b) and 18 out of the 20 reported in red foxes in Asturias were also present in the wolf population, whereas the only two alleles previously detected in Asturian wildlife but absent in the wolf were two private alleles from red fox (Sarms40 and Sarms44, Table 1).

While mites isolated from wolves contained 27 of the 29 alleles previously detected in S. scabiei mites derived from sympatric ungulates and red fox from Asturias, only 3 of these 29 alleles were present both in red fox and ungulate derived mites. This fact allowed the consideration of 9 (out of 12) and 17 (out of 20) alleles as "exclusive" for ungulate species and red fox derived mites respectively, before including $C$. lupus mites in the study.

There was no evidence of linkage disequilibrium for any of the loci examined $(P>0.05)$. The analysis of molecular data showed deviation from Hardy-Weinberg equilibrium (HWE) in all the 9 microsatellite loci studied in wolf mites, the highest value from studied wildlife species in Asturias (HWE had been detected in two and six loci from Pyrenean chamois and red fox respectively, Alasaad et al., 2011b).

Several mite specimens were collected from each of 4 wolves, with intra-host variation detected in three of them. Variation was reported in all loci with the exception of Sarms44. Sarms36 showed variation in the 3 wolves where intra-host variation was detected, and Sarms34, Sarms37 and Sarms38 presented variation in two wolves.

Mites isolated from wolves offered the highest mean number of alleles $(3.111 \pm 0.782)$ from the four studied wild mammal species in Asturias, while herbivore-derived populations showed a lower mean number of alleles $(0.444 \pm 0.882$ in Pyrenean chamois and $0.667 \pm 1.000$ in red deer) than red fox-derived mites $(2.000 \pm 1.225)$ (Table 2).

Mean expected heterozygosity was $0.049 \pm 0.097$ for chamois, $0.117 \pm 0.181$ for red deer, $0.416 \pm 0.232$ for red fox and $0.571 \pm 0.140$ for wolf.

The Bayesian assignment test of the software STRUCTURE, $\ln \operatorname{Pr}(X \mid K)$ for the likely number of populations, indicated $K=2$ as the uppermost cluster value (Fig. 1). This differentiation of two clusters as the best fit grouped

Table2

Number of alleles detected in mites belonging to the four sympatric species studied in Asturias.

\begin{tabular}{|c|c|c|c|c|c|c|c|}
\hline Locus \# & Rupic. 2010 & Cervus & Vulpes & Canis & Mean & s.d. & Total number \\
\hline Sarms33 & 1 & 1 & 2 & 3 & 1.750 & 0.957 & 3 \\
\hline Sarms34 & 2 & 2 & 1 & 3 & 2.000 & 0.816 & 3 \\
\hline Sarms35 & 1 & 1 & 3 & 4 & 2.250 & 1.500 & 4 \\
\hline Sarms36 & 1 & 2 & 3 & 3 & 2.250 & 0.957 & 3 \\
\hline Sarms37 & 1 & 1 & 3 & 4 & 2.250 & 1.500 & 4 \\
\hline Sarms38 & 2 & 2 & 2 & 4 & 2.500 & 1.000 & 4 \\
\hline Sarms40 & 1 & 1 & 2 & 2 & 1.500 & 0.577 & 3 \\
\hline Sarms41 & 1 & 1 & 1 & 2 & 1.250 & 0.500 & 2 \\
\hline Sarms44 & 1 & 1 & 3 & 3 & 2.000 & 1.555 & 4 \\
\hline Mean & 0.444 & 0.667 & 2.000 & 3.111 & 1.556 & 1.244 & 3.333 \\
\hline s.d. & 0.882 & 1.000 & 1.225 & 0.782 & 0.972 & 0.191 & 0.667 \\
\hline
\end{tabular}

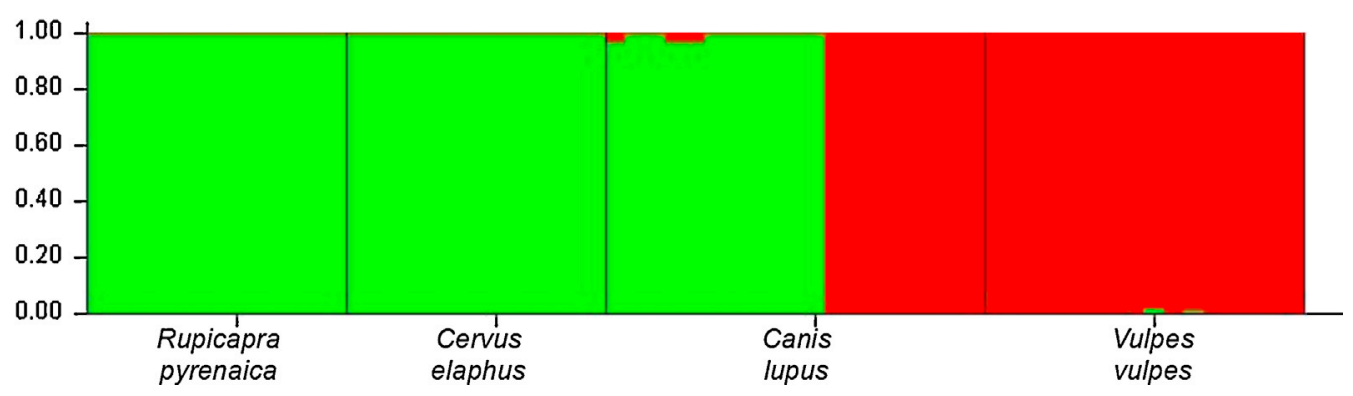

Fig.2. Bar plot of the degree of individual variation between $58 \mathrm{~S}$. scabiei from different host species in Asturias (Spain) assigned to given genetic clusters in STRUCTURE, when two $(K=2)$ populations are assumed in the dataset. Each cluster is represented by a different color. (For interpretation of the references to color in this figure caption, the reader is referred to the web version of the article.) 
Table3

Matrix of fixation index (FST) significant $P$ values, with significance level $P=0.05$ (above diagonal), and population pairwise FST (below diagonal) for each pairwise comparison of four Sarcoptes mite populations from Asturias, Spain.

\begin{tabular}{llll}
\hline & Rupicapra pyrenaica & Cervus elaphus & Canis lupus \\
\hline Rupicapra pyrenaica & & 0.081 & $<0.001^{*}$ \\
Cervus elaphus & 0.118 & & $<0.001^{*}$ \\
Vulpes vulpes & 0.742 & 0.709 & $<0.001^{*}$ \\
Canis lupus & 0.374 & 0.332 & $<0.001^{*}$ \\
\hline
\end{tabular}

Significance level $p=0.05$.

Sarcoptes mites from all herbivore hosts in one cluster, while Sarcoptes mites from red fox formed another cluster and wolves showed mites similar to one or the other cluster (Fig. 2).

AMOVA analysis showed differentiation among populations (FST $=0.48352 ; P<0.001$ ), signaling that the mite component populations differed greatly. The wolf-derived Sarcoptes mite population was statistically $(P<0.001)$ different from those living in the two studied herbivore species (chamois and red deer) and from red-fox derived Sarcoptes mite populations. Herbivore-derived mite populations formed only one group $(P=0.081)$. There were statistically supported $(P<0.001)$ genetic differentiations between the other species-derived Sarcoptes mite populations. FST between wolf derived- and herbivore-derived Sarcoptes mite populations was lower than that between fox derived- and herbivore-derived Sarcoptes mite populations (Table 3).

The scatter plot of the Factorial Component Analysis (FCA) for the individual mites collected on chamois, red deer, red fox and wolf, agreed with the results obtained by the Bayesian assignment test: while red deer and chamois derived mites were similar to each other and appeared grouped, red fox mites gathered separately, and mites collected on wolves were grouped in part with the ungulate-derived mites and in part with the red foxderived mites (Fig. 3).

\section{Discussion}

The present study reveals that wolf mites show the highest number of different alleles $(n=28)$ out of the four wild species surveyed ( $n=20$ alleles in red fox, with none of the ungulate species housing mites with more than 12 detected alleles [Alasaad et al., 2011b - Table 1]). Wolf mites contained 27 of the 29 alleles previously detected in $S$. scabiei originating from sympatric ungulates and red fox in Asturias, thus explaining the low number of private alleles reported in this work and suggesting limited genetic separation and relatively high gene flow between Sarcoptes mite populations when including wolf in the analysis. The higher variability reported in C. lupus mites agrees with results dealing with the mean number of alleles, deviation from Hardy-Weinberg equilibrium (HWE), intra-host variation and mean expected heterozygosity, all of them showing in wolf mites the highest values found in the four analyzed host species. Regarding the genetic diversity among the different Sarcoptes mite populations, the obtained FST values are to be considered high, but they are similar to those obtained in previous studies on S. scabiei (e.g. Gakuya et al., 2011). We have no clear explanation of such high FST values, however the questionable taxonomic status of this parasite could be behind this high FST values, and hence further molecular studies with wider range of molecular markers are needed.

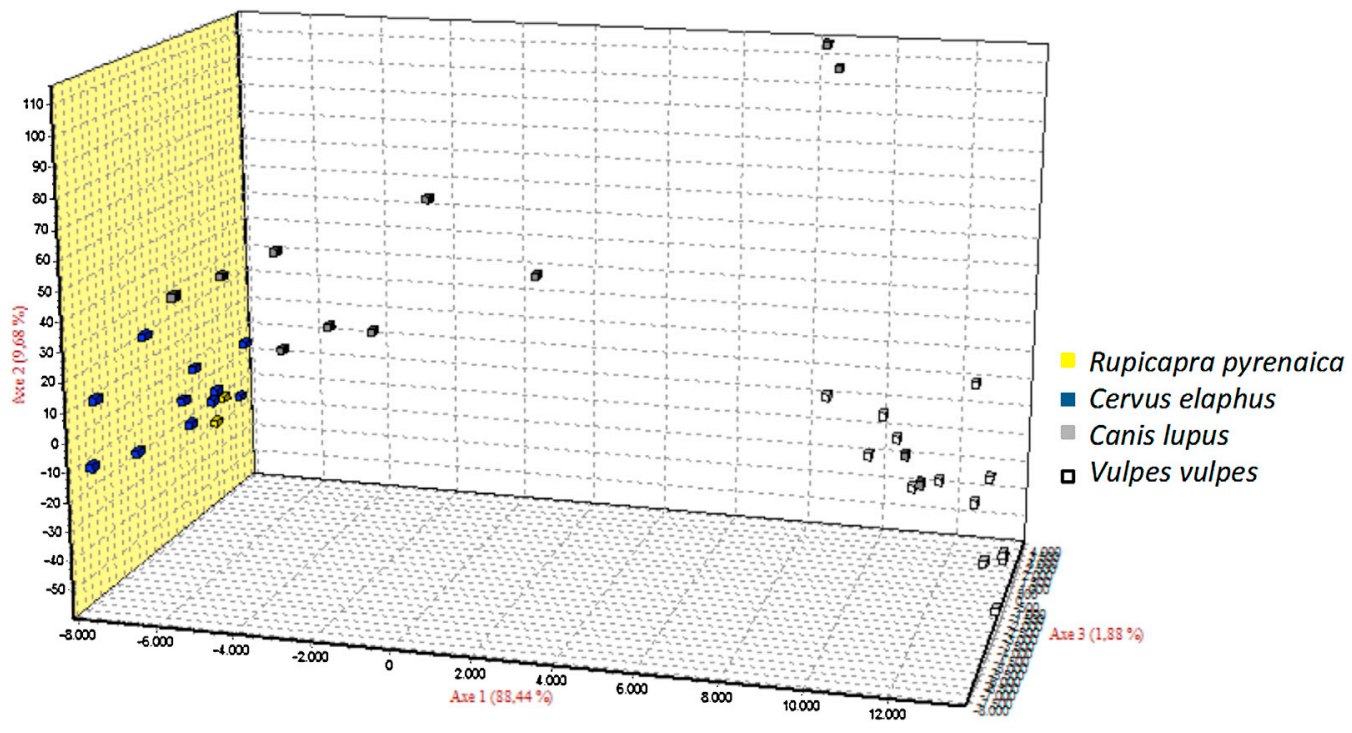

Fig.3. Factorial Component Analysis (FCA) of the proportion of variation of Sarcoptes mite populations of red deer, chamois, red fox and wolf from Asturias (Northern Spain) assigned to given genetic clusters in Genetix. (For interpretation of the references to color in this figure caption, the reader is referred to the web version of the article.) 
In the Masai Mara (Kenya) ecosystem (Gakuya et al., 2011), the genetic study of mites belonging to different species revealed "prey-to-predator" Sarcoptes gene flow leading to deviations from the host taxon phenomenon formulated by Rasero et al. (2010). This work is the first one, in Europe, in which a large predator and the corresponding main sylvatic prey are included in a study aimed to investigate the molecular diversity and gene flow of Sarcoptes mites. As in the case of other large predators in Masai Mara, the inclusion of wolf permitted to investigate the outcome of the enhanced opportunities of Sarcoptes mite transmission between host taxa within a consolidated "predator/prey" chain, with the eventual evasion of the genetic separation of host-associated mites. In Gakuya's work (2011) the "favorite prey" effect led to the identification of four different clusters and highlighted inconsistencies with the host-taxon law. As an interesting difference, in our case data showed the maintenance of the two previously described host-associated clusters (red fox vs. herbivore derived mite populations, Figs. 1-3, Alasaad et al., 2011b) and an apparently milder "prey-to-predator" effect associated with the presence of wolf, whose mites belonged to both clusters with scarce variations. The molecular results in the present work can be better assessed when considering FCA information (showing mites distributed within those from red fox or ungulates, Fig. 3) and the Bayesian assignment test for the likely number of populations $K$ (with $K=2$ as the uppermost cluster value, Fig. 1). These data confirm a substantial compliance with the "host taxon law", with maintenance of a differentiation of mites in two populations (ungulate and red fox derived), and the appearance in wolf of mites belonging to one of these two recognized clusters in Asturias (thus revealing certain "prey to predator" effect). The bar plot representing the degree of individual variation between $S$. scabiei from the different studied host species (Fig. 2) agrees with this view of wolf being able to "collect" mites both from red fox and from ungulates.

In the "prey-to-predator" Sarcoptes gene flow reported in wolves in Asturias, two main differences with the Masai Mara ecosystem results can be found: (i) a more evident "carnivore to carnivore" (red fox-wolf) gene flow than observed between lion and cheetah and (ii) an apparent lack of real gene flow, "evolution" or mixture between red fox- and herbivore-derived mite populations, even though wolves may harbor mites belonging to both. The first difference is probably related to the different ecological role that the red fox plays compared to the wolf, lion or cheetah, implying a different nature and frequency of contacts between wolf and red fox in comparison with the two African predators. On the other hand, further research is necessary to clarify the reasons for the apparent lack of gene admixture and scarce "variation-evolution" suggested by molecular data in wolf derived mites.

It is the Authors' opinion that peculiarities of the immune response developed by the wolf against invading Sarcoptes mites may be a key in the understanding of reported molecular data. As shown by Oleaga et al. (2012), in scabietic wolves the hypersensitivity-associated alopecic form is prevalent on the aneargy-associated hyperkeratotic form which is usually observed in ungulates and red fox. Demographic and necropsy data suggest that this predator is able to exert a certain control over infestation with S. scabiei (Oleaga et al., 2011), in contrast with the usually deadly development of scabies in the other studied sympatric species in Asturias (Oleaga et al., 2012). The apparent efficiency of the immune system of the wolf against mite proliferation may suggest (i) the development of a limited number of mite generations on it and (ii) the possibility of repeated infestations with mites from any of the sympatric species this canid may get into contact (as suggested by the detection, in the present work, of one wolf infested by both "ungulate-like" and "red-fox-like" mites). These two features may hamper host adaptation and facilitate gene flow among mite populations, thus preventing genetic selection and favoring the high variability reported in C. lupus derived mites. A similar situation has been suggested in wild boars, which show greater resistance to these parasites than other mammalian species and whose mites showed high variability and heterozygosity parameters (Rasero et al., 2010). In the case of wolf, the wide range of geographical movement and the large number of species this canid can get into contact (both as predator and as scavenger) are additional arguments favoring repeated infestations with genetically distinct mites from different species.

To complement this type of study it would be interesting to obtain mites isolated from sympatric domestic animals, such as dogs or goats as a possible source of sarcoptic mange for ungulates and even wolf in Asturias. The implementation of tools like the Sarcoptes-World Molecular Network (Sarcoptes- WMN, Alasaad et al., 2011a) can improve collaboration between researchers and allow more profound studies in the future.

Taking into account these and previous results dealing with hosts (namely wild boars) which have not been considered in this study (Rasero et al., 2010), it seems necessary focus on the pathobiology of the mites and the efficiency of the immune response developed by the different host species, as key aspects for a better interpretation and tuning of the "host-taxon law" when studying gene flow and evolution of parasitic mite populations. On the other hand, the role that host species play in studied ecosystems, especially when including susceptible carnivores displaying preying behavior on mangy prey, must be also considered for a correct evaluation of a possible "prey-to-predator" effect. The consideration of mites and their hosts as an ecosystem in constant change and adaptation process requires due attention not only to the biology, ecology and behavior of host species, but also to their immune system performance and the possibly host species population-specific variability of clinical presentations of sarcoptic mange.

\section{Acknowledgements}

This is a contribution to the agreement between CSIC and Principado de Asturias. The research was partially supported by RNM-6400, Proyecto de Excelencia (Junta de Andalucia, Spain), Project RTA 2011-00087-00-00 (INIA), and Juan de la Cierva Grant to Dr S. Alasaad. We thank the rangers of the game preserves (especially Jaime Marcos Beltrán and Francisco Alonso Mier) for their help in carcass 
submission and our colleagues from IREC and SERIDA (Óscar Rodríguez, María Suárez and Alberto Espí) for their assistance in necropsy and laboratory work. We wish to thank also Kevin Dalton for revising the English of the manuscript.

\section{References}

Alasaad, S., Rossi, L., Maione, S., Sartore, S., Soriguer, R.C., Pérez, J.M., Rasero, R., Zhu, X.Q., Soglia, D., 2008a. HotSHOT Plus ThermalSHOCK, a new and efficient technique for preparation of PCR-quality Sarcoptes mite genomic DNA. Parasitol. Res. 103, 1455-1457.

Alasaad, S., Soglia, D., Sarasa, M., Soriguer, R.C., Pérez, J.M., Granados, J.E., Rasero, R., Zhu, X.Q., Rossi, L., 2008b. Skin-scale genetic structure of Sarcoptes scabiei populations from individual hosts: empirical evidence from Iberian ibex derived mites. Parasitol. Res. 104, 101-105.

Alasaad, S., Rossi, L., Soriguer, R.C., Rambozzi, L., Soglia, D., Pérez, J.M., Zhu, X.Q., 2009. Sarcoptes mite from collection to DNA extraction: the lost realm of the neglected parasite. Parasitol. Res. 104, 723-732.

Alasaad, S., Walton, S., Rossi, L., Bornstein, S., Abu-Madi, M., Soriguer, R.C., Fitzgerald, S., Zhu, X.Q., Zimmermann, W., Ugbomoiko, U.S., Pei, K.J.C., Heukelbach, J., 2011a. Sarcoptes-World Molecular Network (Sarcoptes- WMN): integrating research on scabies. Int. J. Infect. Dis. 15 (5), E294-E297.

Alasaad, S., Oleaga, A., Casais, R., Rossi, L., Molinar-Min, A., Soriguer, R.C., Gortázar, C., 2011b. Temporal stability in the genetic structure of Sarcoptes scabiei under the host-taxon law: empirical evidences from wildlife derived Sarcoptes mite in Asturias, Spain. Parasit. Vectors 4, 151.

Alasaad, S., Schuster, R.K., Gakuya, F., Theneyan, H., Jowers, M.J., Maione, S., Molinar-Min, A., Soriguer, R.C., Rossi, L., 2012. Applicability of molecular markers to determine parasitic infection origins in the animal trade: a case study from Sarcoptes mites in wildebeest. Forensic Sci. Med. Pathol. 8, 280-284.

Andrews, J.R.H., 1983. The origin and evolution of host associations of Sarcoptes scabiei and the subfamily Sarcoptinae Murray. Acarologia 24, 85-94.

Barja, I., 2009. Prey and prey-age preference by the Iberian wolf Canis lupus signatus in a multiple-prey ecosystem. Wildl. Biol. 15, 147-154.

Belkhir, K., 1999. GENETIX, logiciel sous WindowsTM pour la génétique des populations, Laboratoire Génome et Populations, Université de Montpellier II, http://www.univmontp2.fr/_genetix

Bornstein, S., Zakrisson, G., Thebo, P., 1995. Clinical picture and antibody response to experimental Sarcoptes scabiei var vulpes infection in red foxes (Vulpes vulpes). Acta Vet. Scand. 36, 509-519.

Bornstein, S., Mörner, T., Samuel, W.M., 2001. Sarcoptes scabiei and sarcoptic mange. In: Samuel, W.M., Pybus, M.J., Kocan, A.A. (Eds.), Parasitic Diseases of Wild Mammals. , 2nd ed. Iowa State University Press, Ames, pp. 107-119.

Domínguez, G., Espí, A., Prieto, J.M., de la Torre, J.A., 2008. Sarcoptic mange in Iberian wolves (Canis lupus signatus) in northern Spain. Vet. Rec. $162,754-755$.

Evanno, G., Regnaut, S., Goudet, J., 2005. Detecting the number of clusters of individuals using the software STRUCTURE: a simulation study. Mol. Ecol. 14, 2611-2620.

Excoffier, L., 2006. Arlequin 3.11 copyright 2006, CMPG University of Berne, available at http://cmpg.unibe.ch/software/arlequin3

Fain, A., 1978. Epidemiological problems of scabies. Int. J. Dermatol. 17, 20-30.

Fernández-Moran, J., Gomez, S., Ballesteros, F., Quirós, P., Benito, J.J., Feliu, C., Nieto, J.M., 1997. Epizootiology of sarcoptic mange in a population of cantabrian chamois (Rupicapra pyrenaica parva) in Northwestern Spain. Vet. Parasitol. 73, 163-171.

Gakuya, F., Rossi, L., Ombui, J., Maingi, N., Muchemi, G., Ogara, W., Soriguer, R.C., Alasaad, S., 2011. The curse of the prey: Sarcoptes mite molecular analysis reveals potential prey-to-predator parasitic infestation in wild animals from Masai Mara, Kenya. Parasite Vector 4, 193.

Gakuya, F., Ombui, J., Heukelbach, J., Maingi, N., Muchemi, G., Ogara, W., Mijele, D., Alasaad, S., 2012a. Knowledge of Mange among Masai Pastoralists in Kenya. PLoS ONE 7 (8), e43342.

Gakuya, F., Ombui, J., Maingi, N., Muchemi, G., Ogara, W., Soriguer, R.C., Alasaad, S., 2012b. Sarcoptic mange and cheetah conservation in Masai Mara (Kenya): epidemiological study in a wildlife/livestock system. Parasitology 139 (12), 1587-1595.
González-Quirós, P., Solano, S., Silva, P., 2002. Population evolution of cantabrian chamois with sarcoptic mange in centre-eastern Asturias (Northwest Spain). Pirineos 157, 109-201.

$\mathrm{Gu}$, X.B., Yang, G.Y., 2008. A study on the genetic relationship of mites in the genus Sarcoptes (Acari: Sarcoptidae) in China. Int. J. Acarol. 32, 183-190.

Kassen, R., 2002. The experimental evolution of specialists, generalists, and the maintenance of diversity. J. Evol. Biol. 15, 173-190.

Lafferty, K.D., Dobson, A.P., Kuris, A.M., 2006. Parasites dominate food web links. Proc. Natl. Acad. Sci. 103, 11211-11216.

Lavín, S., Ruiz-Bascaran, M., Marco, I., Fondevila, M.D., Ramis, A.J., 2000. Experimental infection of chamois (Rupicapra pyrenaica parva) with Sarcoptes scabiei derived from naturally infected goats. J. Vet. Med. 47, 693-699.

Llaneza, L., Fernández, A., Nores, C., 1996. Dieta del lobo en dos zonas de Asturias (España) que difieren en carga ganadera, Doñana. Acta Vertebrata 23 (2), 201-213.

Luong, L.T., Chapman, E.G., Harwood, J.D., Hudson, P.J., 2013. Linking predator-prey interactions with exposure to a trophically transmitted parasite using PCR-based analyses. Mol. Ecol. 22 (1), 239-248.

Magalhães, S., Forbes, M.R., Skoracka, A., Osakabe, M., Chevillon, C., McCoy, K.D., 2007. Host race formation in the Acari. Exp. Appl. Acarol. $42,225-238$

Menzano, A., Rambozzi, L., Rossi, L., 2007. A severe episode of wildlife derived scabies in domestic goats in Italy. Small Rum. Res. 70, 154-158.

Meriggi, A., Lovari, S., 1996. A review of wolf predation in Southern Europe: does the wolf prefer wild prey to livestock? J. Appl. Ecol. 33, $1561-1571$.

Montesu, M.A., Cottoni, F., Bonomo, G.C., Cestoni, D., 1991. Discoverers of the parasitic origin of scabies. Am. J. Dermatopathol. 13, 425-427.

Mörner, T., Eriksson, H., Brojër, C., Nilsson, K., Uhlhorn, H., Agren, E., Hard, A.F., Segerstad, C., Jansson, D.S., Gavierwiden, D., 2005. Diseases and mortality in free-ranging brown bear (Ursus arctos), gray wolf (Canis lupus), and wolverine (Gulo gulo) in Sweden. J. Wildlife Dis. $41,298-303$.

Oleaga, A., Balseiro, A., Gortázar, C., 2008a. Sarcoptic mange in two roe deer (Capreolus capreolus) from northern Spain. Eur. J. Wildl. Res. 54, 134-137.

Oleaga, A., Casais, R., Gonzalez-Quiros, P., Prieto, M., Gortázar, C., 2008b. Sarcoptic mange in red deer from Spain: improved surveillance or disease emergence? Vet. Parasitol. 154, 103-113.

Oleaga, A., Casais, R., Balseiro, A., Espí, A., Llaneza, L., Hartasánchez, A., Gortázar, C., 2011. New techniques for an old disease: sarcoptic mange in the Iberian wolf. Vet. Parasitol. 181, 255-266.

Oleaga, A., Casais, R., Prieto, J.M., Gortázar, C., Balseiro, A., 2012. Comparative pathological and immunohistochemical features of Sarcoptic mange in five sympatric wildlife species in northern Spain. Eur. J. Wildl. Res. 58, 997-1000.

Oosterhout, C., Hutchinson, W.F., Wills, D.P.M., Shipley, P., 2004. MICROCHECKER: software for identifying and correcting genotyping errors in microsatellite data. Mol. Ecol. Notes 4, 535-538.

Pence, D.B., Castro, S.D., Samuel, W.M., 1975. Variation in the chaetotaxy and denticulation of Sarcoptes scabiei (Acarina: Sarcoptidae) from wild canids. Acarologia 17, 160-165.

Pence, D.B., Ueckerman, E., 2002. Sarcoptic mange in wildlife. Revue Scient. Techn. l'Office Int. des Epizooties 21, 385-398.

Pritchard, J.K., Stephens, M., Donnelly, P., 2000. Inference of population structure using multilocus genotype data. Genetics 155, 945-959.

Rasero, R., Rossi, L., Maione, S., Sacchi, P., Rambozzi, L., Sartore, S., Soriguer, R., Spalenza, V., Alasaad, S., 2010. Host taxon-derived Sarcoptes mites in European wildlife animals, revealed by microsatellite markers. Biol. Cons. 143, 1269-1277.

Raymond, M., Rousset, F., 1995. GENEPOP version 1.2: a population genetics software for exact test and ecumenicism. J. Hered. 86, 248-249.

Sukhdeo, M.V., 2012. Where are the parasites in food webs? Parasite Vectors 5, 239.

Wall, R., Shearer, D., 1997. Veterinary Entomology. Chapman and Hall, London, $439 \mathrm{pp}$.

Walton, S.F., Holt, D.C., Currie, B.J., Kemp, D.J., 2004a. Scabies: new future for a neglected disease. Adv. Parasit. 57, 309-376.

Walton, S.F., Dougall, A., Pizzutto, S., Holt, D., Taplin, D., Arlian, L.G., Morgan, M., Currie, B.J., Kemp, D.J., 2004b. Genetic epidemiology of Sarcoptes scabiei (Acari: Sarcoptidae) in northern Australia. Int. J. Parasitol. 34, 839-849.

Zahler, M., Essig, A., Gothe, R., Rinder, H., 1999. Molecular analyses suggest monospecificity of the genus Sarcoptes (Acari: Sarcoptidae). Int. J. Parasitol. 29, 759-766. 